\title{
El tratamiento con tubos de ventilación no mejoró la calidad de vida en niños con otitis media con derrame
}

"Randomised controlled trial of the effect of ventilacion tubes (grommets) on quality of life at age 1-2 years" Rovers MM, Krabbe PF, et al. Arch Dis Child 2001 Jan;84 (1):45-49

\section{Objetivo}

Comparar el efecto sobre la calidad de vida del tratamiento con Tubo de Ventilación (VT) vs. Conducta Expectante (CE) en los niños de 1-2 años de edad con Otitis Media Efusiva (OME).

\section{Diseño}

Ensayo clínico aleatorizado controlado, multicéntrico, con seguimiento de 12 meses (se evaluó a los 6 y 12 meses)

\section{Lugar}

Se realizó en 13 clínicas otorrinolaroingológicas de Holanda.

\section{Pacientes}

Se seleccionaron niños de 1-2 años de edad con OME diagnosticada luego tres pruebas de rastreo.

\section{Intervención}

Fueron asignados $93(49 \%)$ al grupo de tratamiento con VT y 94 al grupo control con CE $(51 \%)$.

\section{Medición de Resultados Principales}

El análisis se realizó por intención de tratar, y el outcome primario fue observar si mejoraba la calidad de vida en el grupo de la intervención, evaluado por medio del TAIQOL (cuestionario de control de calidad que aborda los componentes físico y emocional) y en Escalas de Erickson (interacción padre- hijo).

\section{Resultados Principales}

Se observó una leve mejoría en la calidad de vida del grupo VT, tanto a los 6 como a los 12 meses, pero esta no fue significativo (con $p=0,22$ y $p=0,94$ respectivamente). Inmediatamente luego de la randomización y por desacuerdo con ésta, se perdieron 19 pacientes ( 4 del grupo VT y 15 del CE) y en el seguimiento hubo 3 pérdidas del grupo VT y 8 en el CE. Además a 10 niños del grupo CE se les colocó tubos de ventilación.

\section{Conclusiones}

En los niños de este grupo etario, el tratamiento con tubos de ventilación en OME no mejora la calidad de vida significativamente comparado con la conducta expectante.

\section{Comentario}

La OME se encuentra asociada a un cierto grado de disminución de la audición y por lo tanto parece lógico pensar que afecte el desarrollo normal de los niños, y que su resolución se traduzca en una mejor calidad de vida, cuestión que intentó demostrar este trabajo. Este estudio intentó comparar la calidad de vida del niño en tratamiento con tubos vs. conducta expectante. Es el primer trabajo sobre este tema en este grupo etario, midieron la calidad de vida a través de un cuestionario general en niños, como el TAIQOL'. La interacción padre e hijo se evaluó por medio de las escalas de Erickson. En todos los diagnósticos de OME se utilizaron las mismas normas del MOMES.

El cuestionario TAIQOL es para niños de 1 á 4 años y lo comple$\tan$ sus padres. Posee 13 dominios de los cuales sólo se utilizaron diez, que se consideraron relacionados con OME, también se realizaron diferentes modificaciones en cuanto a sus escalas

En el análisis tuvieron en cuenta las respuestas emocionales sólo si en el componente físico del TAIQOL se percibía un cambio. La pérdida de 19 participantes luego de la randomización ( 4 del VT y 15 del CE) se tradujo en un disbalance en los scores en la línea de base, por lo que fue necesario ajustar para evitar sesgos. Durante el seguimiento hubo 11 pérdidas ( 3 del VT y 8 de CE) pero sin posibilidad de sesgos ya que no se diferenciaban de los que quedaron en el estudio.

En 10 pacientes del CE se indicaron tubos de ventilación, para evitar sesgos se realizó un análisis de sensibilidad con los dos escenarios posibles (excluyéndolos-incluyéndolos) del grupo VT, no se observaron cambios en los resultados finales.

No se lograron los resultados esperados en este estudio, es decir, no se demostró una diferencia significativa entre los dos grupos con respecto a su calidad de vida, pero sí en cuanto a la eficacia del tratamiento con tubos.

No se observó efecto placebo ni una gran diferencia en calidad de vida en niños con efusión permanente a lo largo del seguimiento vs. los que permanecieron libres de efusión en el mismo período (apoyando la hipótesis que en este grupo etario la OME no se relaciona con calidad de vida), y no se logró identificar subgrupos que se beneficien con los tubos de ventilación por escaso poder.

Conclusion del comentador: EI TAIQOL modificado no fue validado (no se informa su confiabilidad, sensibilidad) lo cual no permite saber si las diferencias no significativas se deben a la utilización de un instrumento no efectivo o a que realmente la OME no se encuentra relacionada con calidad de vida en este grupo etario. Por lo tanto no seria apropiado adoptar conductas o cambiar decisiones terapéuticas basándose en este trabajo.

\section{Dr. Mariano Gallo [ Médico pediatra ]}

